Supporting Information:

\title{
Isotope Effects in the Resonant Inelastic Soft X-ray Scattering Maps of Gas-Phase Methanol
}

\author{
A. Benkert ${ }^{1,2}$, F. Meyer ${ }^{2}$, D. Hauschild ${ }^{2}$, M. Blum ${ }^{3}$, W. Yang ${ }^{4}$, R. G. Wilks ${ }^{5}$, M. Bär ${ }^{3,5,6}$, F. \\ Reinert $^{2}$, C. Heske ${ }^{1,3,7,8}$, and L. Weinhardt ${ }^{1,3,7,8, a)}$ \\ ${ }^{1}$ Institute for Photon Science and Synchrotron Radiation (IPS), Karlsruhe Institute of Technology \\ (KIT), Hermann-v.-Helmholtz-Platz 1, 76344 Eggenstein-Leopoldshafen, Germany \\ ${ }^{2}$ Universität Würzburg, Experimentelle Physik VII, Am Hubland, 97074 Würzburg, Germany \\ ${ }^{3}$ Department of Chemistry and Biochemistry, University of Nevada, Las Vegas (UNLV), 4505 \\ Maryland Parkway, NV 89154-4003, USA \\ ${ }^{4}$ Advanced Light Source, Lawrence Berkeley National Laboratory, 1 Cyclotron Road, Berkeley, \\ CA 94720, USA \\ ${ }^{5}$ Renewable Energy, Helmholtz-Zentrum Berlin für Materialien und Energie GmbH, Hahn- \\ Meitner-Platz 1, 14109 Berlin, Germany \\ ${ }^{6}$ Institut für Physik und Chemie, Brandenburgische Technische Universität Cottbus-Senftenberg, \\ Platz der Deutschen Einheit 1, 03046 Cottbus, Germany \\ ${ }^{7}$ ANKA Synchrotron Radiation Facility, Karlsruhe Institute of Technology (KIT), Hermann-v.- \\ Helmholtz-Platz 1, 76344 Eggenstein-Leopoldshafen, Germany \\ ${ }^{8}$ Institute for Chemical Technology and Polymer Chemistry, Karlsruhe Institute of Technology \\ (KIT), Engesserstr. 18/20, 76128 Karlsruhe, Germany
}



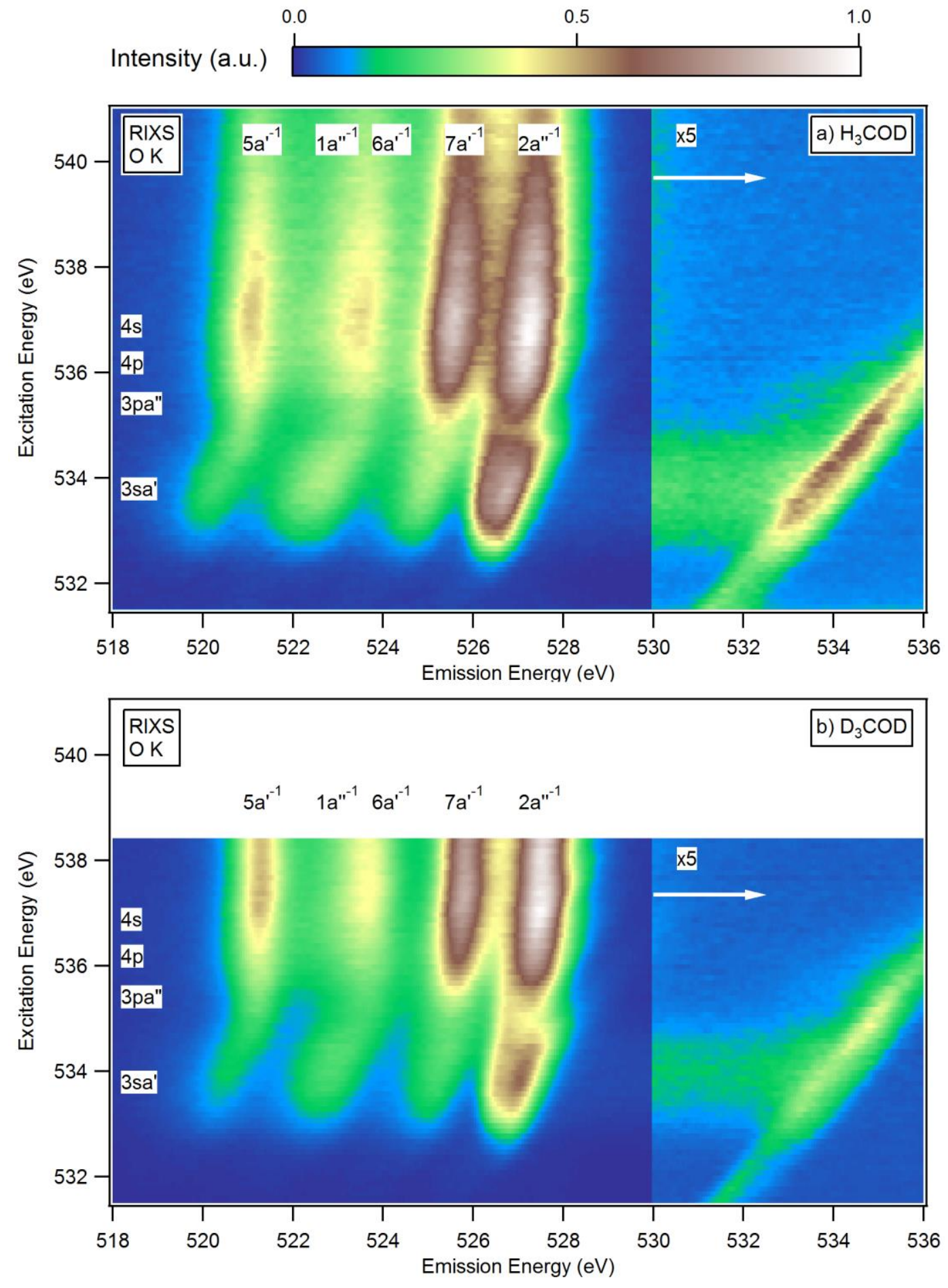

Figure S1: RIXS maps of gas-phase (a) $\mathrm{H}_{3} \mathrm{COD}$ and (b) $\mathrm{D}_{3} \mathrm{COD}$ at the $\mathrm{O} \mathrm{K}$ edge. Spectator emission features and selected absorption resonances are labeled at the top and left, respectively. The participant emission above $530 \mathrm{eV}$ is displayed with a magnified intensity $(\times 5)$. 


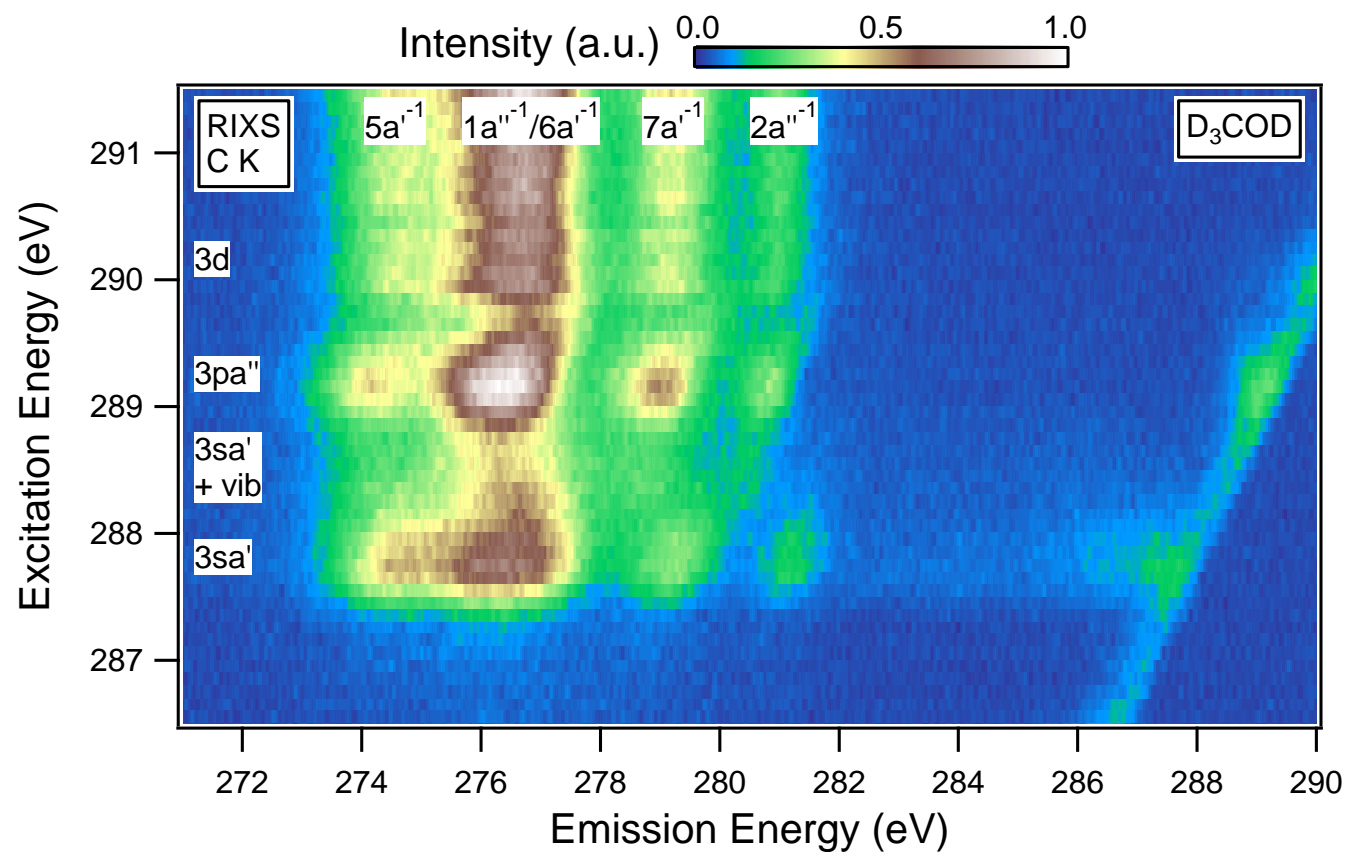

Figure S2: RIXS map of gas-phase $D_{3} C O D$ at the $C K$ edge. Emission features are labeled at the top, selected absorption resonances on the left. 